\title{
Correlated electronic phases in twisted bilayer transition metal dichalcogenides
}

\author{
Lei Wang ${ }^{1,2,11}$, En-Min Shih ${ }^{2,11}$, Augusto Ghiotto 2,11, Lede Xian ${ }^{3}$, Daniel A. Rhodes ${ }^{4}$, Cheng Tan ${ }^{4,5}$, \\ Martin Claassen ${ }^{6}$, Dante M. Kennes ${ }^{3,7}{ }^{3}$, Yusong Bai ${ }^{8}{ }^{8}$, Bumho Kim ${ }^{4}$, Kenji Watanabe ${ }^{9}$, \\ Takashi Taniguchi ${ }^{9}$, Xiaoyang Zhu ${ }^{8}$, James Hone $\mathbb{B}^{4}$, Angel Rubio ${ }^{3,6,10}{ }$, Abhay N. Pasupathy $\mathbb{D}^{2 \bowtie}$ \\ and Cory R. Dean ${ }^{2}{ }^{2 凶}$
}

In narrow electron bands in which the Coulomb interaction energy becomes comparable to the bandwidth, interactions can drive new quantum phases. Such flat bands in twisted graphene-based systems result in correlated insulator, superconducting and topological states. Here we report evidence of low-energy flat bands in twisted bilayer WSe $\mathbf{W}_{2}$ with signatures of collective phases observed over twist angles that range from 4 to $5.1^{\circ}$. At half-band filling, a correlated insulator appeared that is tunable with both twist angle and displacement field. At a $5.1^{\circ}$ twist, zero-resistance pockets were observed on doping away from half filling at temperatures below $3 \mathrm{~K}$, which indicates a possible transition to a superconducting state. The observation of tunable collective phases in a simple band, which hosts only two holes per unit cell at full filling, establishes twisted bilayer transition metal dichalcogenides as an ideal platform to study correlated physics in two dimensions on a triangular lattice.

Tis he advent of van der Waals heterostructures ${ }^{1,2}$ has opened up new avenues to band engineering by simply placing one monolayer on top of another. When the two layers have different lattice constants, or the same lattice constant but are rotated with respect to each other, a long wavelength periodic modulation results, referred to as a moiré superlattice. Coupling to this moiré superlattice in general modifies the electronic band structure ${ }^{3}$ and, in some cases, can result in the formation of low-energy subband ${ }^{4,5}$. Under these circumstances, isolated flat bands have been realized in a wide range of graphene-based structures, which include twisted bilayer graphene $(\mathrm{TBG})^{6-11}$, double bilayer graphene ${ }^{12-15}$ and $\mathrm{ABC}$ trilayer graphene/ $\mathrm{BN}^{16-18}$. Within these flat bands, electron interactions become the dominant energy scale and lead to emergent electronic phases, such as correlated insulators, superconductors, magnetism and topological electronic structures.

In TBG, the flat band appears only within the narrow range of twist angles $1.1 \pm 0.1^{\circ}$, owing to a delicate interplay between the layer hybridization energy and twist-determined band displacements in momentum space ${ }^{4,7,8}$. In heterostructures that combine moiré patterns with semiconductors, the situation is different. Here the resulting moiré miniband extends from the intrinsic band edge to the band energy associated with the moiré Brillouin zone boundary. The bandwidth thus varies continuously with the inverse moire wavelength. Studies of $\mathrm{ABC}$ trilayer graphene/BN and twisted double bilayer graphene exploit this phenomenon by using a transverse displacement field to induce the semiconductor bandgap. However, in both cases the tunability remains limited-in twisted double bilayer graphene, there effectively remains a narrowly defined magic angle, owing to additional displacement-field effects on the overall band structure ${ }^{12-15}$, whereas in $\mathrm{ABC}$ trilayer graphene/BN, the maximal moire wavelength is fixed by the graphene-BN lattice mismatch. Trigonal prismatic transition metal dichalcogenides (TMDs) provide several potential advantages in this regard. As in TBG, theoretical work on TMD homo- and heterobilayers predicts the existence of flat bands and van Hove singularities (vHSs) in the moiré Brillouin zone ${ }^{19-22}$ that could support emergent electronic phases $^{19,20,23}$. In this case, the bandwidth varies continuously with twist angle, without the need for additional displacement fields, and therefore could be widely tunable. Moreover, the reduced degeneracy in TMDs compared to that in graphene, due to coupled spin and valley degrees of freedom, together with material properties not present in graphene, such as strong spin-orbit coupling, suggest that twisted bilayer TMDs could provide an idealized solid-state version of a single-band Hubbard model on a triangular lattice, in which several correlated states may be realized ${ }^{19,24-27}$.

In this work we investigated flat bands that appear in twisted homo-bilayer $\mathrm{WSe}_{2}(\mathrm{tWSe})_{2}$. Our devices were fabricated using the same 'tear-and-stack' method as used to fabricate TBG with a controlled interlayer twist angle ${ }^{2,7,28}$. An illustration of the device structure is shown in Fig. 1a. In brief (see Methods for details), our devices were assembled from high-quality $\mathrm{WSe}_{2}$ material, synthesized by the flux-growth method ${ }^{29}$, and employ prepatterned Hall-bar shaped Pt electrodes ${ }^{30}$ together with a dual-gate structure to achieve ohmic contacts at low temperatures ${ }^{31}$. The twist angles of the measured devices were determined by a combination of techniques, which included atomic force microscopy, optically measured second harmonic generation (SHG) (Supplementary Fig. 18) and transport signatures.

'National Laboratory of Solid-State Microstructures, School of Physics and Collaborative Innovation Center of Advanced Microstructures, Nanjing University, Nanjing, China. ${ }^{2}$ Department of Physics, Columbia University, New York, NY, USA. ${ }^{3}$ Max Planck Institute for the Structure and Dynamics of Matter, Hamburg, Germany. ${ }^{4}$ Department of Mechanical Engineering, Columbia University, New York, NY, USA. ${ }^{5}$ Department of Electrical Engineering, Columbia University, New York, NY, USA. ${ }^{6}$ Center for Computational Quantum Physics, Flatiron Institute, New York, NY, USA. ${ }^{7}$ Institut für Theorie der Statistischen Physik RWTH Aachen University and JARA-Fundamentals of Future Information Technology, Aachen, Germany. ${ }^{8}$ Department of Chemistry, Columbia University, New York, NY, USA. ${ }^{9}$ National Institute for Materials Science, Tsukuba, Ibaraki, Japan. ${ }^{10}$ Nano-Bio Spectroscopy Group, Departamento de Fisica de Materiales, Universidad del País Vasco, San Sebastian, Spain. "'These authors contributed equally: Lei Wang, En-Min Shih and Augusto Ghiotto. 凶e-mail: angel.rubio@mpsd.mpg.de; apn2108@columbia.edu; cdean@phys.columbia.edu 


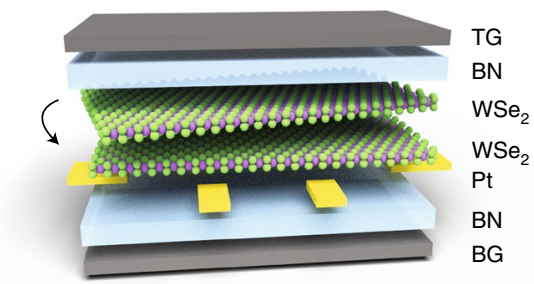

b
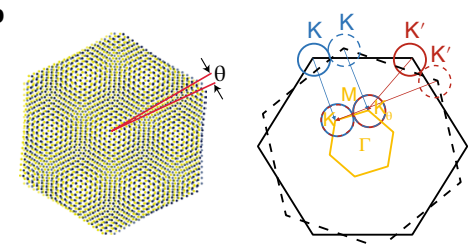

c

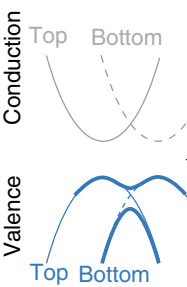

$(\mathrm{K}, \uparrow)$ d

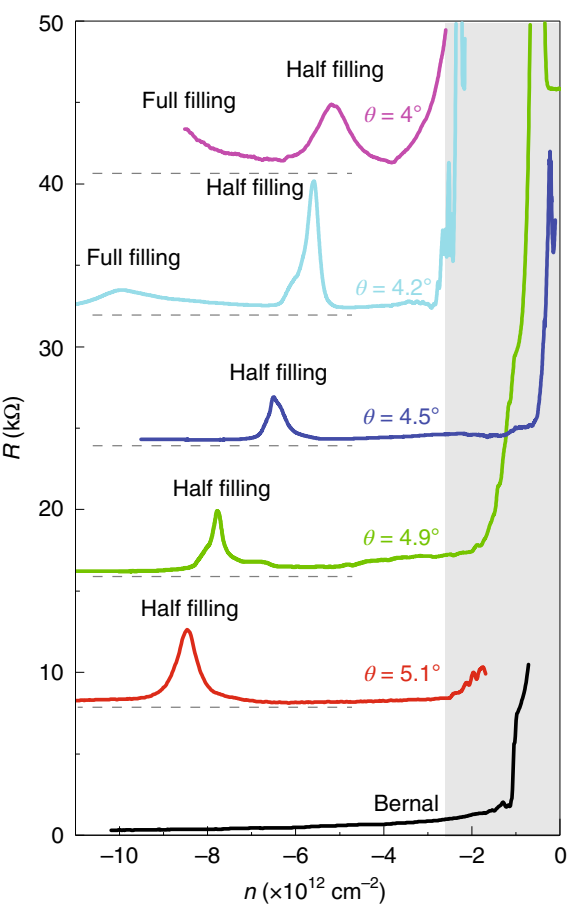

e

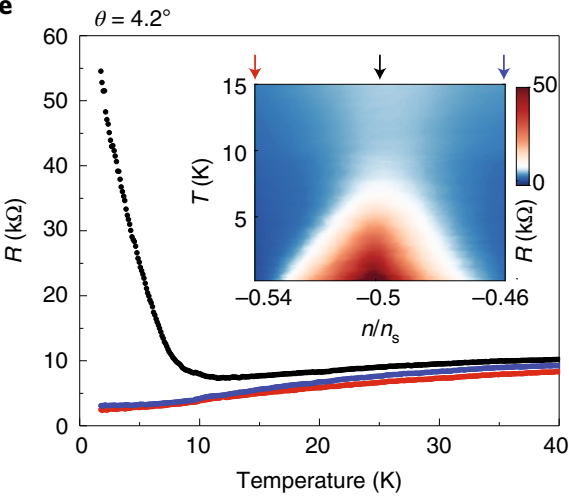

f

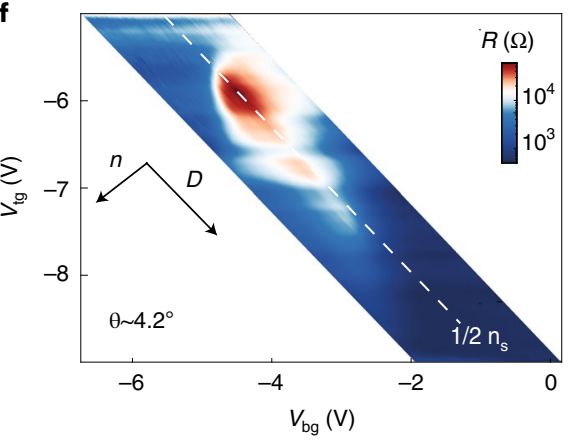

Fig. 1 | Flat bands in twisted bilayer $\mathbf{W S e}_{2}$. $\mathbf{a}$, Schematic representation of the device structure. $\mathbf{b}$, Left: real-space representation of the moiré pattern that results from a small angle twist between the two WSe ${ }_{2}$ layers. Right: Brillouin zones of the top (solid line) and bottom (dashed line) layers. The states near $\mathrm{K}$ and $\mathrm{K}^{\prime}$ are shown as blue and red, respectively. The resultant band structure in the moiré Brillouin zone (yellow) is also displayed. c, Schematic representation of the twisted bilayer WSe ${ }_{2}$ hybridized band structure in the K valley, spin up (left), and $\mathrm{K}^{\prime}$ valley, spin down (right). $\mathbf{d}$, Resistance as a function of carrier density $n$, measured at $T=1.8 \mathrm{~K}$ for six WSe ${ }_{2}$ bilayer configurations. 'Bernal' refers to the natural stacking arrangement (equivalent to a $60^{\circ}$ twist). The curves are offset for clarity. The dashed lines correspond to the zero resistance for each curve. To capture the peak feature at half filling, these curves were measured at different displacement-field values $\left(4^{\circ}, D=0.08 \mathrm{Vnm}^{-1} ; 4.2^{\circ}, D=0.24 \mathrm{Vnm}^{-1} ; 4.5^{\circ}, D=0.19 \mathrm{Vnm}{ }^{-1} ; 4.9^{\circ}, D=0.29 \mathrm{Vnm}^{-1}\right.$; $\left.5.1^{\circ}, D=0.83 \mathrm{~V} \mathrm{~nm}^{-1}\right)$. The grey shaded region roughly denotes the mobility edges of the devices, which might vary among devices due to different disorders introduced in the fabrication process. e, Resistance as a function of temperature from the twist $4.2^{\circ}$ device measured at $D=0.15 \mathrm{~V}$ nm ${ }^{-1}$, which highlights the activation of the Mott gap at half filling of the moiré band and the metallic behaviour at densities away from half filling. Inset: resistance map as a function of temperature and band filling. $\mathbf{f}$, Resistivity map as a function of top gate and back gate voltages ( $V_{\mathrm{tg}}$ and $V_{\mathrm{bg}}$, respectively) measured at $T=1.8 \mathrm{~K}$, showing the evolution of the half-filling resistance with displacement field $D$ (dashed line). Black arrows indicate the direction of increasing $D$ and $n$.

A schematic illustration of the moiré superlattice and resulting band structure for tWSe $e_{2}$ is shown in Fig. 1b,c. In the vicinity of the $\mathrm{K}$ point of the Brillouin zone, the band structure of each $\mathrm{WSe}_{2}$ layer is a single spin-polarized parabolic band. Electron hopping between the layers caused a strong hybridization of the bands where they intersect, which leads to a low-energy miniband (Fig. 1c). For chemical potentials that are close to the valence band edge (as is the case in our samples), the physics of $\mathrm{tWSe}_{2}$ is thus well approximated by a single subband whose width is determined by the twist angle. From angle-dependent band-structure calculations, fitting over the range of $2-7^{\circ}$ (Supplementary Fig. 2), we find that the width of this subband, $w(\mathrm{meV})$, grows approximately as $w \approx 4.5 \theta^{2}-5.4 \theta+7.1$ with the twist angle $\theta$ in degrees. However, estimating the Coulomb interaction to be $U=e^{2} / 4 \pi \epsilon_{0} \epsilon_{\mathrm{r}} L$, where $\epsilon_{\mathrm{r}}$ is the effective dielectric constant in the bilayer and $L$ is the moire wavelength, implies that $U$ increases linearly with the twist angle ${ }^{7}$. Taking $\epsilon_{\mathrm{r}} \approx 10$ (ref. ${ }^{32}$ ) suggests a crossover angle of $\sim 2^{\circ}$ below which (above which) $U>w$ $(U<w)$. However, there are substantial uncertainties in the length scale over which the electron wavefunction is localized within a single moiré site, as well as in the effective dielectric constant. The true crossover angle could easily be a factor of two or more larger than the estimate above (Supplementary Fig. 3). Regardless, the order of magnitude estimate implies that $U$ is comparable to $w$ in the range of twist angles we studied, which probably places us in the interesting regime of moderate correlations.

Figure 1d shows a plot of the resistivity versus hole-band carrier density at $T=1.8 \mathrm{~K}$ for five different twisted $\mathrm{WSe}_{2}$ devices with angles that range from 4 to $5.1^{\circ}$, and also one naturally occurring Bernal bilayer stacking that corresponds to an equivalent twist of $60^{\circ}$. All the devices exhibited a diverging resistance for hole densities less than $\sim 2 \times 10^{12} \mathrm{~cm}^{-2}$, as the Fermi energy approached the intrinsic band edge of $\mathrm{WSe}_{2}$. This sets a lower limit to the density range we can access by transport measurement.

At large densities, we observed new resistive peaks, whose position shifted to a lower density at smaller angles. The moiré unit cell area $A$ relates to the twist angle $\theta$ according to $A=\frac{a^{2} \sqrt{3}}{4(1-\cos \theta)}$, where $a=0.328 \mathrm{~nm}$ is the $\mathrm{WSe}_{2}$ lattice constant ${ }^{33}$. From this we observed that all the devices exhibit a resistive peak that coincides with one hole per moiré unit cell, or half filling of the moiré subband (Supplementary Fig. 4). In addition, within the accessible density range limited by the gate dielectric breakdown, a second resistive peak was seen in the 4.2 and $4^{\circ}$ devices near full filling of the moiré subband (that is, doping to two holes per moiré unit cell).

The appearance of the resistive features confirmed the presence of a low-energy band that results from the twist-induced moiré 

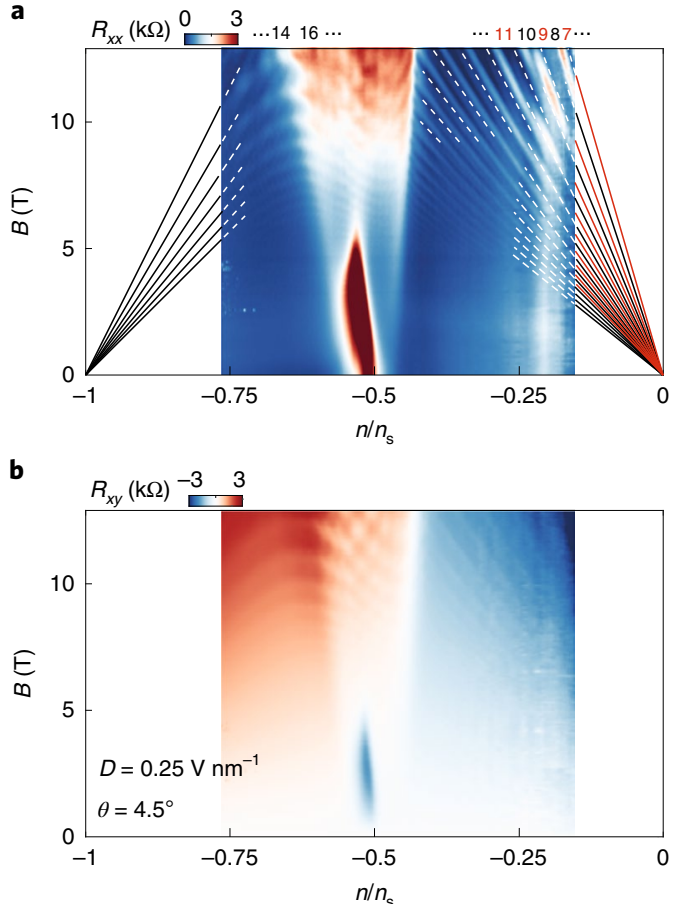

C
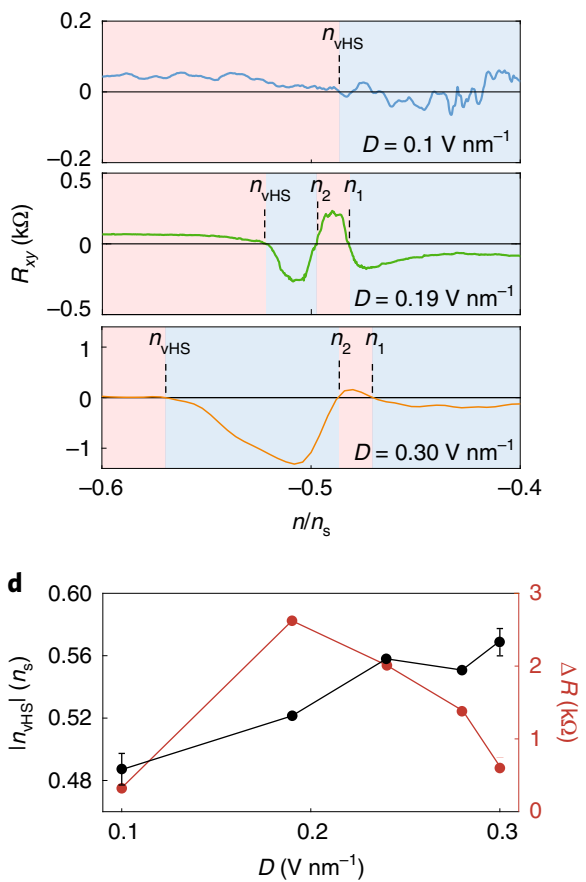

e

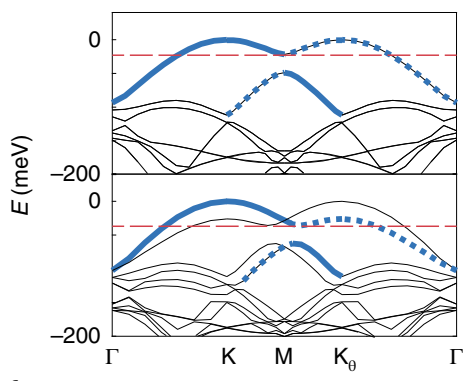

$\mathbf{f}$

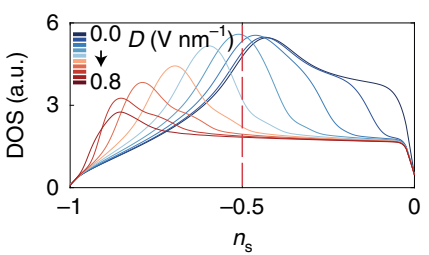

g

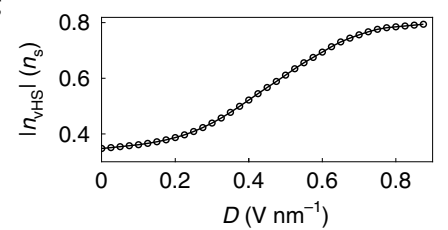

Fig. 2 | Displacement-field dependence of vHS for $4.5^{\circ} \mathrm{tWSe} \mathrm{2}_{2}$. a, Longitudinal resistance, $R_{x x^{\prime}}$ as a function of magnetic field $B$ and band filling $n / n_{\mathrm{s}^{\prime}}$ measured at $D=0.25 \mathrm{~V} \mathrm{~nm}^{-1}$ and $T=1.8 \mathrm{~K}$. Two Landau fans are resolved, which project (solid lines) to the band edge and full filling of the moiré subband. Black and red solid lines identify the even and odd integer quantum Hall states with corresponding filling factors labelled on top of the figure, respectively. The dashed white lines identify the emerging quantum Hall gap on the map. $\mathbf{b}$, Hall resistance, $R_{x y}$ as a function of magnetic field and band filling, measured simultaneously with $R_{x x} . \mathbf{c}, R_{x y}$ as a function of band filling, measured at $B=1 \mathrm{~T}$ for different displacement fields. $n_{\mathrm{vHS}}$ labels the position of the single-particle band vHS for each $D ; n_{1}$ and $n_{2}$ label the additional sign changes that we associate with the correlated insulator. $\mathbf{d}$, Black line: vHS position in terms of band filling versus $D$, determined from the Hall resistance at $B=1 \mathrm{~T}$. Error bars represent the uncertainty of the crossing position, limited by the measurement noise. Red line: increment resistance at half filling versus $D\left(\Delta R=R_{n} / n_{s}=0.5-R_{n} / n_{s}=0.6\right)$. e, Density functional theory calculations of the top valence bands of the moiré Brillouin zone for $D=0 \mathrm{Vnm}^{-1}$ (top) and $D=0.3 \mathrm{Vnm}^{-1}$ (bottom) for a $5.08^{\circ}$ twist angle. We highlight the contributions from the $\mathrm{K}$ valley of the top layer (solid blue line) and from the $\mathrm{K}$ valley of the bottom layer (dashed blue line) to the topmost bands. Red dashed lines indicate the energy level of half filling of the topmost valence band. $\mathbf{f}$, Calculated DOS for the top two valence bands as a function of filling for a $4.5^{\circ}$ twist angle. g, Calculated vHS position versus displacement field for a $4.5^{\circ}$ twist angle.

superlattice $^{19-22}$ (by comparison, the Bernal device showed a featureless response with the resistance monotonically decreasing with increasing doping). The resistance peak near full filling is consistent with a reduction in the density of states (DOS) near the edge of the moiré subband ${ }^{19,21,22}$. In contrast, the resistive state at half filling in the twisted devices is not anticipated in a single-particle band structure, and therefore indicates that the correlations are sufficiently strong within this band to induce a correlation-driven insulating state.

Figure 1e shows the temperature dependence near half filling for the $4.2^{\circ}$ device (Supplementary Fig. 5 for similar data from another device). We define the band filling as $\nu=n / n_{\mathrm{s}}$ where $n$ is the gate-induced hole density and $n_{\mathrm{s}}$ is the density necessary to reach full filling of the moiré band. Above approximately $T_{\mathrm{CI}} \approx 10 \mathrm{~K}$ (the critical temperature at which the insulating response onsets), the sample shows a metallic response at all fillings with the resistance decreasing with decreasing temperature. However, below $10 \mathrm{~K}$ the resistance at $0.5 n_{\mathrm{s}}$ shows a sudden onset of the thermally activated behaviour, which indicates an insulating response. The extracted gap value is $\sim 27 \mathrm{~K}$ (Supplementary Fig. 6), or almost three times $T_{\mathrm{CI}}$. The sharp transition together with an activation energy that exceeds the onset temperature are both consistent with a true phase transition at $T_{\mathrm{CI}}$, rather than a crossover with decreasing temperature.

Figure $1 \mathrm{f}$ shows the resistance of the same $4.2^{\circ}$ device versus the top and back gate voltages (see Supplementary Fig. 7 for other angle data) in the range of half filling, which allowed us to investigate the effect of displacement field-here we report the average displacement field defined as $D=\left(C_{\mathrm{T}} V_{\mathrm{T}}-C_{\mathrm{B}} V_{\mathrm{B}}\right) / 2 \epsilon_{\mathrm{o}}$, where $V_{\mathrm{T}(\mathrm{B})}$ is the top (bottom) gate bias, $C_{\mathrm{T}(\mathrm{B})}$ the associated geometric capacitance and $\epsilon_{\mathrm{o}}$ the vacuum permittivity. We note that the resistive peak at $0.5 n_{\mathrm{s}}$ persists only over a finite range, $0.08 \mathrm{~V} \mathrm{~nm}^{-1}<D<0.28 \mathrm{~V} \mathrm{~nm}^{-1}$, with the maximum resistance observed at $D \approx 0.15 \mathrm{~V} \mathrm{~nm}^{-1}$. Temperature-dependent measurements (Supplementary Fig. 8) confirmed a $D$-field-induced metal-insulator-metal transition over this range, with activation gaps that showed a dome-like evolution.

The response of the samples under perpendicular applied magnetic fields provides key additional insights. We first mapped the Landau level spectra as a function of applied field and induced density to unambiguously determine the density of full filling of the moiré lattice. Next, we measured the Hall response to determine the point at which the band curvature changed from electron like to hole like, which should coincide with a peak (vHS) in the DOS. Figure 2a shows the longitudinal resistance $R_{x x}$ for the $4.5^{\circ}$ device plotted against $n / n_{\mathrm{s}}$ at magnetic fields up to $13 \mathrm{~T}$. At high fields, two sets of Landau fans are visible. Linear extrapolation of the Landau level trajectories to zero field allowed us to precisely determine the position of the valence band edge as well as the full filling density of the moiré subband (see Supplementary Fig. 9 for data from other angle devices). For the fan that originated from the valence band edge, we observed only even-integer quantum Hall states (white lines in Fig. 2a) up to approximately $B=8 \mathrm{~T}$, consistent with a 


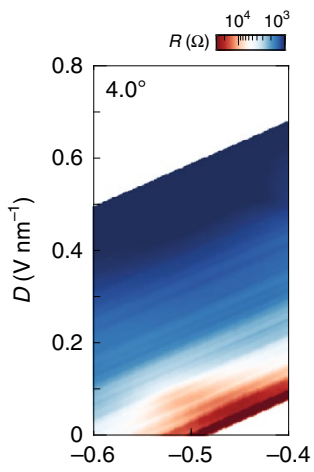

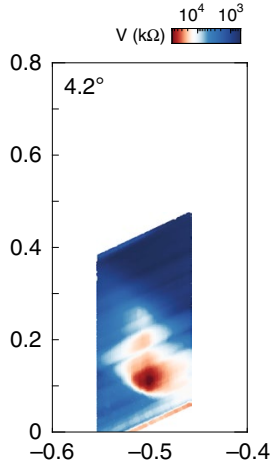

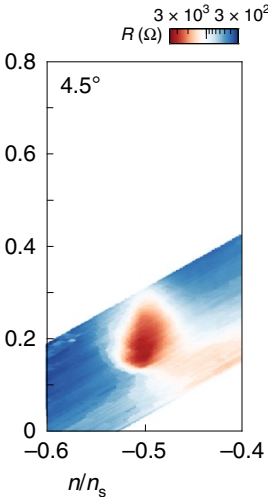

$n / n_{\mathrm{s}}$ b

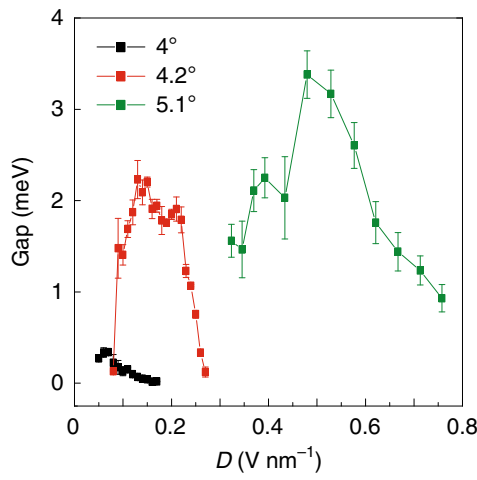

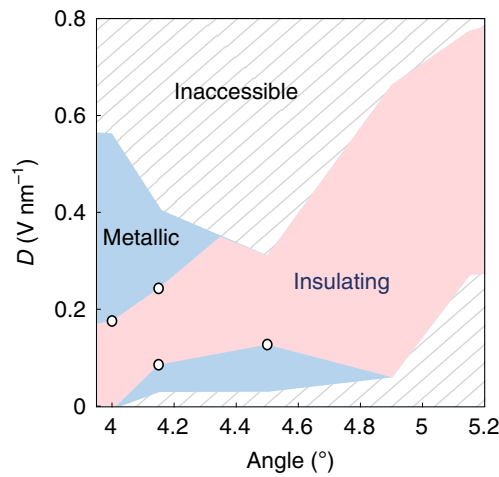
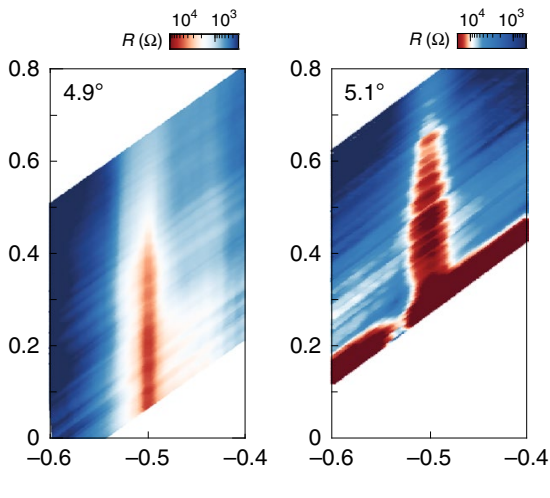

d

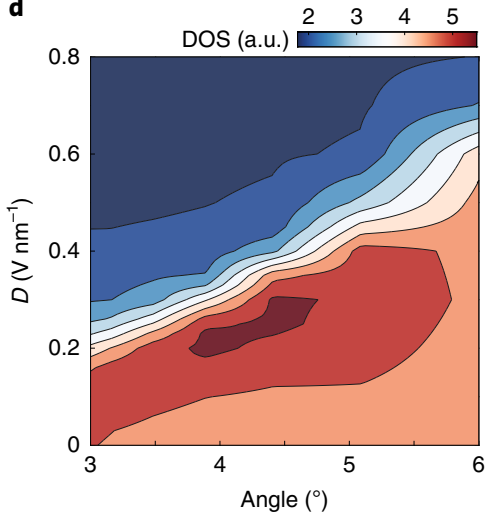

Fig. 3 | Angle and displacement-field dependence of the correlated insulator. a, Resistance versus displacement field and normalized density near half filling for all the measured angles. b, Insulating gap size versus displacement field for devices with $4^{\circ}, 4.2^{\circ}$ and $5.1^{\circ}$ twist angles. Error bars represent the uncertainty that arises from determining the linear (thermally activated) regime for the fit. c, Phase diagram of tWSe ${ }_{2}$ as a function of displacement field and twist angle, showing the observed metallic and insulating regions at half filling. White circles denote the observed metal-to-insulator transitions. d, Calculated DOS at half filling as a function of displacement field and twist angle, showing a qualitative correspondence with c.

twofold degenerate Fermi surface. Above $8 \mathrm{~T}$, we observed the onset of additional odd integer quantum Hall effect states (red lines), which indicates a lifting of the combined spin-valley degeneracy. This degeneracy lifting was not clearly observed in the satellite fan that originated from full filling, in which the twofold degenerate Landau levels persisted to the highest measured fields.

The magnitude of the $0.5 n_{\mathrm{s}}$ insulating peak decreased with increasing $B$ field and vanished at $B \approx 6 \mathrm{~T}$. No change in the insulating state was observed when applying a parallel $B$ field (Supplementary Information Fig. 10). This behaviour is qualitatively consistent with a non-ferromagnetically ordered ground state. However, a detailed understanding of this trend is complicated by the variation of both the Coulomb and Zeeman energies under a perpendicularly applied $D$ field. A full study of the $B$-field dependence is beyond the scope of this article and will be discussed elsewhere (Supplementary Fig. 11).

Figure $2 \mathrm{~b}$ shows the Hall resistance measured that was simultaneously with the longitudinal resistivity shown in Fig. 2a. At a high field, the sign of the Hall resistance inverts on doping from a low to high carrier density, consistent with the expected response for a single band where the dispersion changes from hole like to electron like as the band is filled. This provides further confirmation that our gate range spans a single low-energy subband. Figure $2 \mathrm{c}$ shows the Hall resistance versus density, measured at $B=1 \mathrm{~T}$ for three separate displacement fields. The top panel $\left(D=0.1 \mathrm{~V} \mathrm{~nm}^{-1}\right)$ shows a low displacement field value in which the corresponding longitudinal resistance, $R_{x x}$, does not show a resistivity peak at half filling, which suggests there was no insulating state (Supplementary Fig. 12). Here, the Hall resistance changed sign from hole-like to electron-like transport at $n / n_{\mathrm{s}} \approx-0.46$, after which the Hall effect continued to show electron-like transport with increased doping. As there is no evidence of an insulating state, we interpret the single crossing point as the position of the vHS at which the moiré subband curvature changed sign. The two lower panels of Fig. $2 \mathrm{c}\left(D=0.19 \mathrm{~V} \mathrm{~nm}^{-1}\right.$ (middle) and $D=0.30 \mathrm{~V} \mathrm{~nm}^{-1}$ (bottom)) correspond to the range of $D$ in which a resistive peak in $R_{x x}$ was observed at half filling. In this case, three crossings appear, which we label $n_{1}, n_{2}$ and $n_{\mathrm{vHS}}$. We identify the $n_{\mathrm{vHS}}$ crossing as the single-particle band vHS as this marks the point beyond which the band continues to show an electron-like Hall response. We therefore associate the other two crossings with the appearance of the half-filling insulating state.

The position of the vHS evolved continuously with displacement field, starting at $\left|n / n_{\mathrm{s}}\right|<0.5$ for a low $D$, but moving through half filling to $\left|n / n_{s}\right|>0.5$ values for a large $D$. The magnitude of the longitudinal resistance peak at half filling correlates closely with the position of the single-particle vHS with the largest resistive peak seen when the vHS is near half filling (Fig. 2d). The dependence of the half-filling correlated insulator on the vHS position indicates that the system was in a regime of moderate correlations, in which the band structure (specifically, the DOS) plays an important role in determining the properties of the emergent insulator. However, we note that the insulating phase itself is always observed at precisely half filling, which indicates that we are not in the weakly correlated regime where gaps would form at the position of the vHS.

Figure $2 \mathrm{e}-\mathrm{g}$ shows the tWSe $\mathrm{H}_{2}$ band structure calculated by density functional theory and tight binding as a function of $D$. At zero $D$, the two layers are degenerate and the hybridization of the valence bands results in a vHS exactly at the M point of the band structure (Supplementary Fig. 13). As a displacement field is applied to the 
a

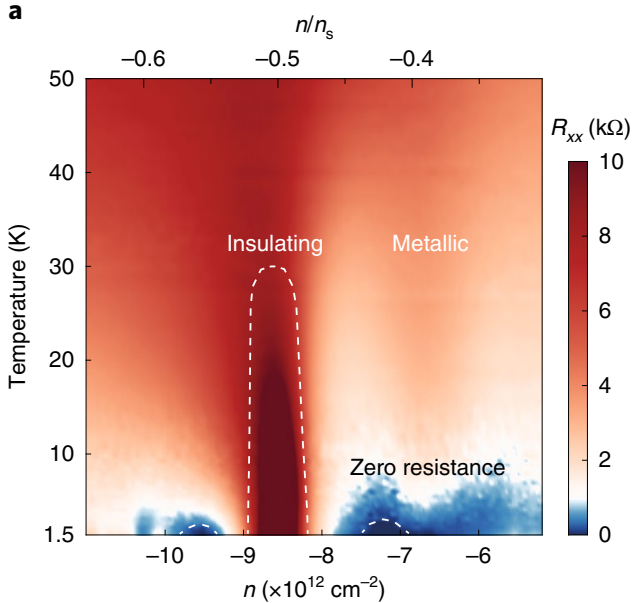

b

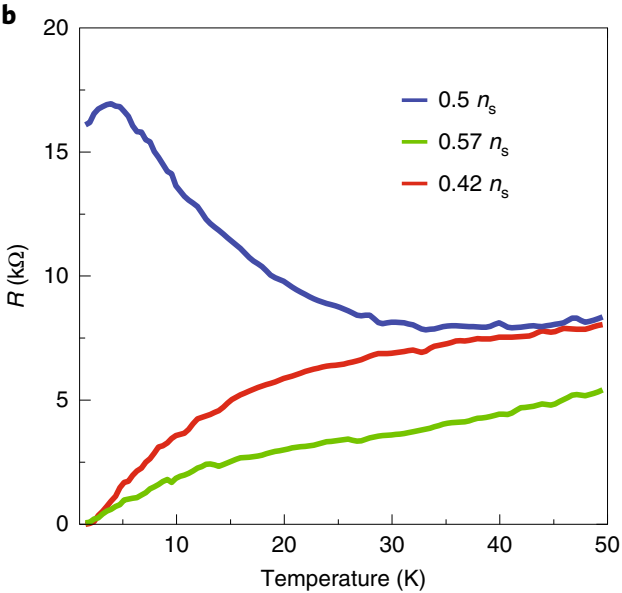

Fig. 4 | Observation of zero resistance regions around half filling. a, Resistance as a function of temperature and density for a twist angle of $5.1^{\circ}$ at $D=0.45 \mathrm{Vnm}^{-1}$. The density is varied with $V_{\mathrm{bg}}$, while $V_{\mathrm{tg}}$ is at $-12.25 \mathrm{~V}$. Two zero-resistance regions, which suggests a transition to superconducting behaviour, appear when doping away from the insulating state. $\mathbf{b}$, Resistance as a function of temperature at densities near half filling. The insulating response at half filling (blue) onsets at $35 \mathrm{~K}$, whereas zero resistance (red and green) is observed at temperatures below $3 \mathrm{~K}$.

sample, the bands from the two layers acquire differing onsite potentials. As a consequence, the position of the vHS shifts away from the $M$ point and down in energy (at extreme displacement fields, such as $1 \mathrm{Vnm}^{-1}$, the two layers become effectively decoupled in the first moiré subband, but, in practice, we did not reach such fields in the experiments). Figure $2 \mathrm{f}$ shows the calculated DOS over the whole moiré subband. At $D=0$, the vHS (peak DOS) is located at slightly less than half filling. With increasing $\mathrm{D}$, the vHS shifted through half filling and quickly moved towards full filling at a large field (Fig. 2g). This trend is qualitatively consistent with our understanding of the Hall response under varying $D$ (Fig. 2c,d), as discussed above. The DOS at half filling varied non-monotonically with $D$, initially increasing slightly and then dropping to about a third of the maximum value at larger fields (Supplementary Fig. 14). The correspondence between this trend and the non-monotonic evolution of the resistive peak with $D$ (Fig. 1f) further supports our interpretation that the strength of the insulator strongly depends on the DOS.

Figure $3 \mathrm{a}$ summarizes the behaviour of the correlated insulator for every twist angle we measured. We observed a displacement-field-driven metal-to-insulator transition at half filling for $4,4.2$ and $4.5^{\circ}$ twists, whereas for 4.9 and $5.1^{\circ}$ the insulator-metal transition at a low displacement field was not observed due to the limitations on the achievable gate voltages. The evolution of the correlated insulator gap versus displacement field, determined from thermal activation, is shown in Fig. $3 b$ (see Supplementary Fig. 15 for the Arrhenius plots). For the 4.2 and $5.1^{\circ}$ devices, we confirmed a non-monotonic gap evolution, which peaked at a non-zero $D$ value. From these plots, we constructed a phase diagram for $\mathrm{tWSe}_{2}$ as a function of angle and displacement field, shown in Fig. 3c. Red identifies the region of insulating behaviour, blue identifies metallic and 'inaccessible' indicates the high (low) displacement field range in which the dielectric leakage exceeded $1 \mathrm{nA}$ (contacts are non-ohmic). Note that the emergence of the insulator at half filling shifted to a larger displacement field with increasing angle. Figure $3 \mathrm{~d}$ shows the DOS at half filling from single-particle band-structure calculations. A qualitative correlation is seen between the two plots, which suggests that the DOS at half filling, as analysed for the $4.5^{\circ}$ device (Fig. 2), plays an important role in stabilizing over the entire angle range we measured.

We next speculate on the nature of the insulating phase in this system. Our data clearly shows the presence of a vHS in the band. In the limit of weak electronic correlations, we could expect a symmetry-breaking order to emerge when the chemical potential is placed at the peak in the DOS. However, in the experiment, we always found the insulating phase at half filling, which indicates that the weak coupling scenario is not valid. However, in the limit of strong correlations, the natural candidate for such an insulating phase is the Mott insulator, in which double occupancy of a given moiré site is forbidden. In this limit, however, the insulating phase becomes insensitive to the details of the band structure, unlike the result we see here (Supplementary Figs. 7 and 8). The situation we have is between both of these limits. One possibility is that we are still in a Mott insulating phase, but simply in a situation in which the correlations are comparable to the bandwidth, that is, near the metal-insulator boundary ${ }^{34}$. Other possibilities include nesting-driven instabilities that are pinned to half filling by the shape of the Fermi surface or Wigner crystal states ${ }^{35}$, in which the state is based on the collective real-space arrangement of holes. Based on transport alone, we are unable at present to distinguish between these possibilities, and future experimental work, which will possibly include spectroscopic and local imaging, may provide answers to this question.

Finally, we investigated whether correlations in doped tWSe ${ }_{2}$ can lead to the emergence of superconductivity $y^{6,8,10,12,14,16}$. Figure 4 shows the temperature dependence of the $5.1^{\circ}$ sample in the vicinity of half filling. These data were acquired at $D=0.45 \mathrm{~V} \mathrm{~nm}^{-1}$, which corresponds to the condition of strongest correlated insulator observed for any of the devices measured (Fig. 3b). The resistance was measured using the smallest currents we could source in practice $(5 \mathrm{nA})$ at this displacement field. On either side of the correlated insulator state, we observed regions in which the resistance reached zero within our instrumentation noise of $\sim 10 \Omega$, which suggests a potential superconducting transition. Figure $4 \mathrm{~b}$ shows the resistivity versus $T$ at half filling, in which an insulator behaviour is seen at low $T$, as well as at two densities where the zero-resistance state is observed. For all three densities, the temperature dependence showed a linear-in- $T$ metallic behaviour above $\sim 40 \mathrm{~K}$. At half filling, thermally activated behaviour onset below $35 \mathrm{~K}$, whereas at the densities where zero resistance was observed, the resistance began to deviate from its high temperature linear slope at around $15 \mathrm{~K}$, dropping finally to zero at around $3 \mathrm{~K}$ (Supplementary Fig. 16). In this device, the zero-resistance state appeared to be fragile and was unstable to repeated thermal cycling. Similar zero-resistance behaviour was observed in a $4.8^{\circ}$ device (Supplementary Fig. 17). In this case, current-voltage measurements showed a transition from a linear behaviour at high temperature to a non-linear behaviour at low temperature, consistent with a superconductor transition, but the 
superconductor gap appears only weakly formed. For other angles, we also observed non-linear drops in resistance in the vicinity of half filling, suggestive of incipient superconductivity, but saturating to non-zero values (Supplementary Fig. 5). These behaviours are all consistent with mesoscopic inhomogeneities in the samples, similar to those reported in graphitic systems ${ }^{7,8,18}$; however, we note that at present we cannot rule out the possibility that the low-temperature phase represents a transition to some other low resistance, but non-superconducting, ground state.

In conclusion, we demonstrate the ability to engineer flat bands in tWSe $e_{2}$ over a range of twist angles. We show that this system hosts correlation-driven emergent phenomena similar to graphitic moiré systems, but with very different degeneracies and single-particle band structures. Owing to their widely tunable electronic structure, this provides a model system for exploring other emergent electronic phases, such as exciton condensates, spin liquids and magnetic ordering. More generally the system provides a tunable solid-state realization of a single-band Hubbard model on a triangular system in which the bandwidth and doping can be independently varied, which allows opportunities to study the interplay between strong interactions and frustration.

\section{Online content}

Any methods, additional references, Nature Research reporting summaries, source data, extended data, supplementary information, acknowledgements, peer review information; details of author contributions and competing interests; and statements of data and code availability are available at https://doi.org/10.1038/ s41563-020-0708-6.

Received: 22 December 2019; Accepted: 11 May 2020; Published online: 22 June 2020

\section{References}

1. Dean, C. R. et al. Boron nitride substrates for high-quality graphene electronics. Nat. Nanotechnol. 5, 722-726 (2010).

2. Wang, L. et al. One-dimensional electrical contact to a two-dimensional material. Science 342, 614-617 (2013).

3. Park, C.-H., Yang, L. I., Son, Y.-W., Cohen, M. L. \& Louie, S. G. Anisotropic behaviours of massless Dirac fermions in graphene under periodic potentials. Nat. Phys. 4, 213-217 (2008).

4. Bistritzer, R. \& MacDonald, A. H. Moiré bands in twisted double-layer graphene. Proc. Natl Acad. Sci. USA 108, 12233-12237 (2011).

5. SuárezMorell, E., Correa, J. D., Vargas, P., Pacheco, M. \& Barticevic, Z Flat bands in slightly twisted bilayer graphene: tight-binding calculations. Phys. Rev. B 82, 121407 (2010).

6. Cao, Y. et al. Unconventional superconductivity in magic-angle graphene superlattices. Nature 556, 43-50 (2018).

7. Cao, Y. et al. Correlated insulator behaviour at half-filling in magic-angle graphene superlattices. Nature 556, 80-84 (2018).

8. Yankowitz, M. et al. Tuning superconductivity in twisted bilayer graphene. Science 363, 1059-1064 (2019).

9. Sharpe, A. L. et al. Emergent ferromagnetism near three-quarters filling in twisted bilayer graphene. Science 365, 605-608 (2019).

10. Lu, X. et al. Superconductors, orbital magnets and correlated states in magic-angle bilayer graphene. Nature 574, 653-657 (2019).
11. Serlin, M. et al. Intrinsic quantized anomalous Hall effect in a moiré heterostructure. Science 367, 900-903 (2020).

12. Liu, X. et al. (2019) Spin-polarized correlated insulator and superconductor in twisted double bilayer graphene. Preprint at: http://arxiv.org/ abs/1903.08130 (2019).

13. Cao, Yuan, et al. Tunable correlated states and spin-polarized phases in twisted bilayer-bilayer graphene. Nature https://doi.org/10.1038/s41586-0202260-6 (2020).

14. Shen, C. et al. Correlated states in twisted double bilayer graphene. Nat. Phys. 16, 520-525 (2020).

15. Burg, G. W. et al. Correlated insulating states in twisted double bilayer graphene. Phys. Rev. Lett. 123, 197702 (2019).

16. Chen, G. et al. Evidence of a gate-tunable Mott insulator in a trilayer graphene moiré superlattice. Nat. Phys. 15, 237-241 (2019).

17. Chen, G. et al. Signatures of tunable superconductivity in a trilayer graphene moiré superlattice. Nature 572, 215-219 (2019).

18. Chen, G. et al. Tunable correlated Chern insulator and ferromagnetism in a moiré superlattice. Nature 579, 56-61 (2020).

19. Wu, F., Lovorn, T., Tutuc, E. \& Macdonald, A. H. Hubbard model physics in transition metal dichalcogenide moiré bands. Phys. Rev. Lett. 121, 26402 (2018)

20. Wu, F., Lovorn, T., Tutuc, E., Martin, I. \& Macdonald, A. H. Topological insulators in twisted transition metal dichalcogenide homobilayers. Phys. Rev. Lett. 122, 86402 (2019).

21. Naik, M. H. \& Jain, M. Ultraflatbands and shear solitons in moiré patterns of twisted bilayer transition metal dichalcogenides. Phys. Rev. Lett. 121, 266401 (2018).

22. Ruiz-Tijerina, D. A. \& Fal'Ko, V. I. Interlayer hybridization and moiré superlattice minibands for electrons and excitons in heterobilayers of transition-metal dichalcogenides. Phys. Rev. B 99, 125424 (2019).

23. Schrade, C. \& Fu, L. Spin-valley density wave in moiré materials. Phys. Rev. B 100, 035413 (2019).

24. Dagotto, E. Correlated electrons in high-temperature superconductors. Rev. Mod. Phys. 66, 763-840 (1994).

25. Scalapino, D. J. A common thread: the pairing interaction for unconventional superconductors. Rev. Mod. Phys. 84, 1383-1417 (2012).

26. LeBlanc, J. P. F. et al. Solutions of the two-dimensional Hubbard model: benchmarks and results from a wide range of numerical algorithms. Phys. Rev. X 5, 041041 (2015).

27. Mazurenko, A. et al. A cold-atom Fermi-Hubbard antiferromagnet. Nature 545, 462-466 (2017).

28. Kim, K. et al. van der Waals heterostructures with high accuracy rotational alignment. Nano Lett. 16, 1989-1995 (2016).

29. Edelberg, D. et al. Approaching the intrinsic limit in transition metal diselenides via point defect control. Nano Lett. 19, 4371-4379 (2019).

30. Movva, H. C. P. et al. High-mobility holes in dual-gated $\mathrm{WSe}_{2}$ field-effect transistors. ACS Nano 9, 10402-10410 (2015).

31. Movva, H. C. P. et al. Density-dependent quantum Hall states and Zeeman splitting in monolayer and bilayer WSe $e_{2}$. Phys. Rev. Lett. 118, 247701 (2017).

32. Laturia, A., Van de Put, M. L. \& Vandenberghe, W. G. Dielectric properties of hexagonal boron nitride and transition metal dichalcogenides: from monolayer to bulk. npj 2D Mater. Appl. 2, 6 (2018).

33. Kormányos, A. et al. k.p theory for two-dimensional transition metal dichalcogenide semiconductors. 2D Materials 2, 022001 (2015).

34. Imada, M., Fujimori, A. \& Tokura, Y. Metal-insulator transitions. Rev. Mod. Phys. 70, 1039-1263 (1998).

35. Padhi, B., Setty, C. \& Phillips, P. W. Doped twisted bilayer graphene near magic angles: proximity to Wigner crystallization, not Mott insulation. Nano Lett. 18, 6175-6181 (2018).

Publisher's note Springer Nature remains neutral with regard to jurisdictional claims in published maps and institutional affiliations.

(C) The Author(s), under exclusive licence to Springer Nature Limited 2020 


\section{Methods}

Device fabrication. Hall-bar-shaped $\mathrm{Cr} / \mathrm{Pt}(2 \mathrm{~nm} / 20 \mathrm{~nm})$ contacts were evaporated on the top of hexagonal boron nitride (hBN) (30-70 nm) $\left(\right.$ ref. $\left.{ }^{30}\right)$. Using a thin polypropylene carbonate film sitting on top of a polydimethylsiloxane microlens, another hBN $(30-70 \mathrm{~nm})$ was then picked up using the dry stamp method ${ }^{2}$. The $\mathrm{hBN}$ was then used to tear part of an exfoliated monolayer $\mathrm{WSe}_{2}$ using the same tear-and-stack method as that used to fabricate TBG with a controlled interlayer twist angle ${ }^{7,28}$. The resulting stack was then dropped onto the prepatterned contacts ${ }^{30}$. The twist angles of the measured devices were first confirmed by SHG measurements and atomic force microscopy. A metal gate was then evaporated on top of the Hall-bar channel. For the back gate of our devices we used a variety of materials, namely $\mathrm{Si}, \mathrm{Cr} / \mathrm{Pd}$ $(2 \mathrm{~nm} / 10 \mathrm{~nm})$ or thin graphite $(10 \mathrm{~nm})$, that were preassembled using the same dry-stamp technique before evaporating the prepatterned contacts onto the bottom hBN (see Supplementary Fig. 18).

SHG measurements. The crystal orientation of the $\mathrm{WSe}_{2}$ monolayers was determined by SHG measurements on an inverted microscope (Olympus IX73) system. Linearly polarized femtosecond laser light $(80 \mathrm{MHz}, 800 \mathrm{~nm}, 100 \mathrm{fs}$, Coherent Mira 900) was focused onto a monolayer with a $\times 100$, numerical aperture 0.80 objective (Olympus LMPLFLN100X). The reflected $400 \mathrm{~nm} \mathrm{SHG}$ signals were collected by the same objective, filtered by a shortpass dichroic mirror, shortpass and bandpass filters and a Glan-Taylor linear polarizer, detected by a photomultiplier tube (Hamamatsu R4220P) and counted by a photon counter (Stanford Research Systems SR400). The SHG experiment was performed by rotating either the sample or the laser polarization (by rotating a half waveplate) with a fixed-polarization detection. Owing to the $D_{3 h}$ symmetry, the non-vanishing tensor elements of the second-order susceptibility of $\mathrm{WSe}_{2}$ monolayers are $\chi_{y y y}^{(2)}=\chi_{y x x}^{(2)}=\chi_{x x y}^{(2)}=\chi_{x y x}^{(2)}$ where the $x$ axis is along the zigzag direction. When rotating the sample, the SHG intensity showed a six-fold symmetry: $I_{\perp} \propto \cos ^{2}(3 \theta)$ and $I_{\|} \propto \sin ^{2}(3 \theta)$, where $\theta$ is the angle between the laser polarization and the zigzag direction. When rotating the laser polarization, the SHG intensity showed a four-fold symmetry: $I_{y} \propto \cos ^{2}(2 \theta)$ and $I_{x} \propto \sin ^{2}(2 \theta)$. Monolayer $\mathrm{WS}_{2}$ flakes grown by chemical vapour deposition in which the zigzag directions that were the same as the crystal edges were used to calibrate the SHG set-up (see Supplementary Fig. 18 for the schematics and data).

\section{Data availability}

The data that support the findings of this study are available from the corresponding author upon request.

\section{Acknowledgements}

Studies of the tunable correlated states in the twisted bilayer WSe ${ }_{2}$ were supported as part of Programmable Quantum Materials, an Energy Frontier Research Center funded by the US Department of Energy (DOE), Office of Science, Basic Energy Sciences (BES), under award DE-SC0019443. The synthesis of the $\mathrm{WSe}_{2}$ crystals was supported by the NSF MRSEC programme through Columbia in the Center for Precision Assembly of Superstratic and Superatomic Solids (DMR-1420634). The theoretical work was supported by the European Research Council (ERC-2015-AdG694097), cluster of Excellence AIM, SFB925 and Grupos Consolidados (IT1249-19). The Flatiron Institute is a division of the Simons Foundation. We acknowledge support from the Max Planck-New York Center for Non-Equilibrium Quantum Phenomena. We thank F. Wu and L. Fu for helpful discussions. We also thank O. Stapleton, P. Wu and Z. Zheng for help in the device fabrication.

\section{Author contributions}

L.W., E.-M.S., A.G., C.T. and D.A.R. fabricated the samples. L.W., E.-M.S. and A.G. performed the transport measurements and analysed the data. Y.B. and X.Z. performed the SHG measurements. D.A.R., B.K. and J.H. grew the $\mathrm{WSe}_{2}$ crystals. K.W. and T.T. grew the hBN crystals. L.X. performed the density functional theory and tight-binding calculations. M.C. and D.M.K. did the mean-field calculations. A.R. supervised the theoretical aspects of this work. L.W., E.-M.S., A.G., C.R.D. and A.P. wrote the manuscript with input from all the authors.

\section{Competing interests}

The authors declare no competing interest.

\section{Additional information}

Supplementary information is available for this paper at https://doi.org/10.1038/ s41563-020-0708-6.

Correspondence and requests for materials should be addressed to A.R., A.N.P. or C.R.D Reprints and permissions information is available at www.nature.com/reprints. 